\title{
From dull to shiny: A novel setup for reflectance difference analysis under catalytic conditions
}

Willem G. Onderwaater, Andriy Taranovskyy, G. Marien Bremmer, Gertjan C. van Baarle, Joost W. M. Frenken, and Irene M. N. Groot

Citation: Review of Scientific Instruments 88, 023704 (2017); doi: 10.1063/1.4975930

View online: $h t t p: / / d x . d o i . o r g / 10.1063 / 1.4975930$

View Table of Contents: http://aip.scitation.org/toc/rsi/88/2

Published by the American Institute of Physics

\section{Articles you may be interested in}

Low vibration laboratory with a single-stage vibration isolation for microscopy applications

Review of Scientific Instruments 88, 023703 (2017); 10.1063/1.4975832

Development of X-ray CCD camera based X-ray micro-CT system

Review of Scientific Instruments 88, 023702 (2017); 10.1063/1.4975376

A needle probe to detect surface enhanced Raman scattering (SERS) within solid specimen

Review of Scientific Instruments 88, 023107 (2017); 10.1063/1.4975183

A simple and versatile phase detector for heterodyne interferometers

Review of Scientific Instruments 88, 023504 (2017); 10.1063/1.4975992

Combined scanning probe microscopy and $x$-ray scattering instrument for in situ catalysis investigations Review of Scientific Instruments 87, 113705 (2016); 10.1063/1.4968804

Backreflection diagnostics for ultra-intense laser plasma experiments based on frequency resolved optical gating

Review of Scientific Instruments 88, 023503 (2017); 10.1063/1.4975827

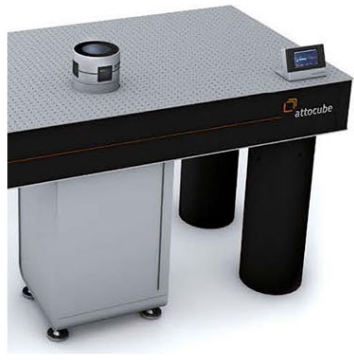

Obstruction free access

optical table with integrated cryocooler

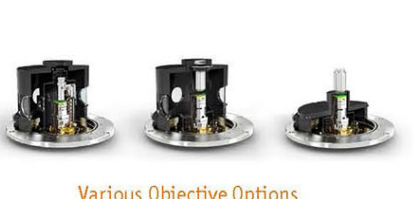

Various Objective Options

\section{attoDRY800}

- Cryogenic Temperatures

- Ultra-Low Vibration

- Optical Table Included

- Fast Cooldown

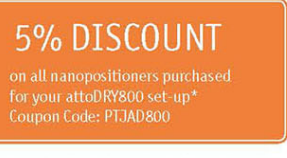




\title{
From dull to shiny: A novel setup for reflectance difference analysis under catalytic conditions
}

\author{
Willem G. Onderwaater, ${ }^{1,2}$ Andriy Taranovskyy, ${ }^{1} \mathrm{G}$. Marien Bremmer, ${ }^{1}$ \\ Gertjan C. van Baarle, ${ }^{3}$ Joost W. M. Frenken, ${ }^{1, a)}$ and Irene M. N. Groot ${ }^{1,4, b)}$ \\ ${ }^{1}$ Huygens-Kamerlingh Onnes Laboratory, Leiden University, P.O. Box 9504, 2300 RA Leiden, \\ The Netherlands \\ ${ }^{2}$ European Synchrotron Radiation Facility, BP 220, F-38043 Grenoble Cedex 9, France \\ ${ }^{3}$ Leiden Probe Microscopy B.V., J.H. Oortweg 19, 2333 CH Leiden, The Netherlands \\ ${ }^{4}$ Leiden Institute of Chemistry, Leiden University, P.O. Box 9502, 2300 RA Leiden, The Netherlands
}

(Received 8 November 2016; accepted 26 January 2017; published online 14 February 2017)

\begin{abstract}
We have developed an experimental setup for optically monitoring a catalytically active surface under reaction conditions. A flow reactor with optical access allows us to image the behavior of an active catalyst surface down to the millimeter length scale. We use reflectance difference measurements with $625 \mathrm{~nm}$ light to investigate $\mathrm{CO}$ oxidation on $\mathrm{Pd}(100)$ at $300 \mathrm{mbar}$ and $320^{\circ} \mathrm{C}$. We conclude that the changes in visible contrast result from the formation of an oxide layer after surface oxidation. Published by AIP Publishing. [http://dx.doi.org/10.1063/1.4975930]
\end{abstract}

\section{INTRODUCTION}

The last two decades have brought us an exciting new "look" on heterogeneous catalysis. Where the catalyst surfaces were originally thought of as mere, two-dimensional playgrounds for the adsorbing and reacting molecules and their products, we now know that these surfaces are intimately involved in the chemical transitions, to the point that they can be heavily affected by the exposure to high temperature and to the high partial pressures of the reactants. The high temperature alone can already lead to a roughening of metal surfaces, ${ }^{1,2}$ thus spontaneously introducing atomic-height steps that can act as efficient binding and reaction sites. The high reactant concentrations can stabilize adsorption structures that would be energetically unfavorable at low pressures. In this way, at sufficiently high pressures, even completely new surface structures can be formed, such as the surface oxides that have been found on various metal surfaces that serve as oxidation catalysts. $^{3}$

Most of these changes have been followed from nanoscopic to rather microscopic scales, using, for example, surface X-ray diffraction (SXRD) and high-pressure scanning tunneling microscopy (STM). ${ }^{4-8}$ As a typical example, we refer to the $\operatorname{Pd}(100)$ surface. It has been shown that during the $\mathrm{CO}$ oxidation reaction on this palladium surface, a surface oxide can be formed. ${ }^{4-7,9,10}$ In the STM images, it can be seen that when this surface oxide is formed, the surface is becoming increasingly rough during the reaction. ${ }^{4,9,10}$ This roughening effect has been ascribed to the result of a Mars-van Krevelen-type reaction mechanism. ${ }^{11}$

Even though the small-scale features of the CO oxidation reaction on $\operatorname{Pd}(100)$ have been observed by STM, and the oxide structure has been resolved with X-ray methods,

\footnotetext{
a) Current address: Advanced Research Center for Nanolithography, P.O. Box 93019, 1090 BA Amsterdam, The Netherlands.

b) Electronic mail: i.m.n.groot@lic.leidenuniv.nl
}

some observations could not be explained by either technique. These observations could find their origin in the spatial inhomogeneity of the reaction over the sample surface. During the catalysis experiment, the 1-cm-diameter palladium surface is located inside a flow reactor. The reactant gases are fed into the reactor at a controlled flow rate and the total pressure is set by controlling the pressure at the outlet of the reactor. It has been shown that in the reactor geometry used for the SXRD experiments, the high turnover frequency of the $\mathrm{CO}$ oxidation reaction causes lateral variations in the gas composition at the sample surface. ${ }^{12}$ In our reactor geometry, this results in a lower impingement rate of $\mathrm{CO}$ molecules in the center of our crystal than at the edge of the sample, near the gas inlet. Such differences in local behavior can be missed by STM, when investigating a relatively small surface area, or by X-ray diffraction methods, where often the beam footprint covers a large area, so that only a spatial average is being recorded. Clearly, it is necessary to inspect the reaction with a field of view that is large enough to cover the entire surface and with a spatial resolution that makes it possible to follow different parts of the surface independently, ideally down to the field of view of the other microscopic tools, which would be close to $1 \mu \mathrm{m}$ for STM.

Techniques such as photoemission electron microscopy have shown remarkable spatio-temporal patterns on a catalyst during CO-oxidation at $5 \times 10^{-4}$ mbar as described by Nettesheim et al. ${ }^{13}$ The features they describe have a micrometer length scale, but they clearly show the spatial and temporal features of the catalyst reaction to behave in complex ways. However, this technique is not suitable for the higher pressures used in industrial catalysis.

Reflectance difference measurements, where the change in reflectivity is measured in real time during some surfacealtering process, have been used successfully in the past to monitor very subtle surface processes. The non-invasiveness of the technique and the fact that light is only weakly scattered at ambient gas pressures has led to the use of optical 
tools such as reflectance difference measurements, reflection anisotropy microscopy, and ellipsometry for crystal growth ${ }^{14,15}$ and catalysis processes. ${ }^{16-18}$ Although the diagnostic power of reflectance difference spectroscopy is greatly enhanced by the use of multiple wavelengths, much of the relevant surface behavior can be inferred already by using a single wavelength, i.e., monochromatic light. Surface anisotropy, when the surface shows different characteristics along its in-plane axes, can be explored by investigating the response of the two different polarization directions. In view of the square symmetry of $\operatorname{Pd}(100)$, however, we expect the surface characteristics to be identical in all in-plane directions.

In previous experiments, we had noticed that after exposure of $\mathrm{Pd}(100)$ to $\mathrm{CO}$ oxidation conditions, the surface had changed visibly. It had turned from an almost perfect mirror into a slightly dulled mirror. The origin of this transition was unknown since our setups do not provide optical access to the sample in its reactor environment. In order to analyze and quantify these optical changes during the catalytic reaction, we built a new experimental setup, using simple optics. The aim of this setup is to image the optical reflectance of a complete, 1-cm-diameter model catalyst sample, with high temporal resolution. We are interested in both the specularly and the diffusely scattered light from the sample surface. Resolving the angular intensity dependence of the diffusely scattered light, which is related to the characteristic length scales of the surface, would enable us to study the possible existence of mirror planes on the rough surface, which might be relevant information for the catalytic process.
This paper describes the design of this setup and presents the findings of the first experiments. We investigate the origins of the contrast changes during the $\mathrm{CO}$ oxidation reaction on $\operatorname{Pd}(100)$.

\section{METHODS}

We measure the sample reflectance with a home-built reflectometer. The sample is housed in a small flow reactor, designed by the company Leiden Probe Microscopy B.V. (LPM), shown in Figure 1. This mini-reactor is a simplified version of the ReactorSXRD chamber, ${ }^{19}$ with the same sample mounting stage and similar reactor volume but without the UHV sample preparation environment. The chosen geometry enables a direct comparison of the optical data with the SXRD results obtained previously. With an LPM gas supply system, we can set the total flow rate, total pressure (120 mbar2000 mbar (the latter is chosen as an upper limit because of safety specifications of the reactor window)), and partial pressure ratios between all constituent gases at ratios ranging from 100:1 up to 1:100. The maximum flow rate per constituent gas is $10 \mathrm{ml}_{n} / \mathrm{min}$. With a reactor volume of $\sim 16 \mathrm{ml}$, the refresh rates are in the order of $1 \mathrm{~min}$. This has to be taken into account when switching the gas composition in the reactor. The composition of the gas mixture that leaves the reactor is measured with an LPM high pressure inlet residual gas analyzer, T100 residual gas analyzer. ${ }^{20}$ The reflectometer itself consists of two stages. The pre-sample stage starts with a light source. A collimator collects the light and a spatial filter creates a parallel beam. The post-sample stage collects the light, chooses a specific imaging mode, and records the result with a camera.

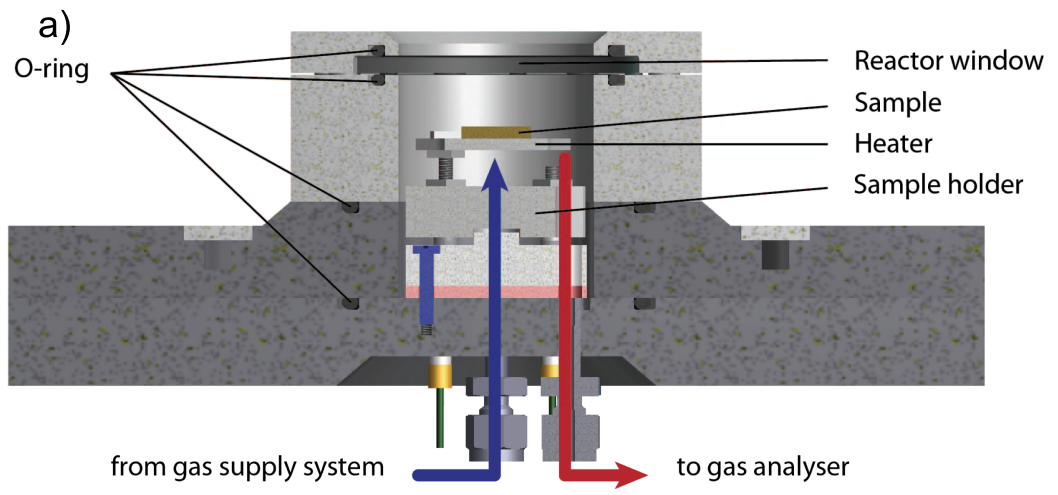

b)

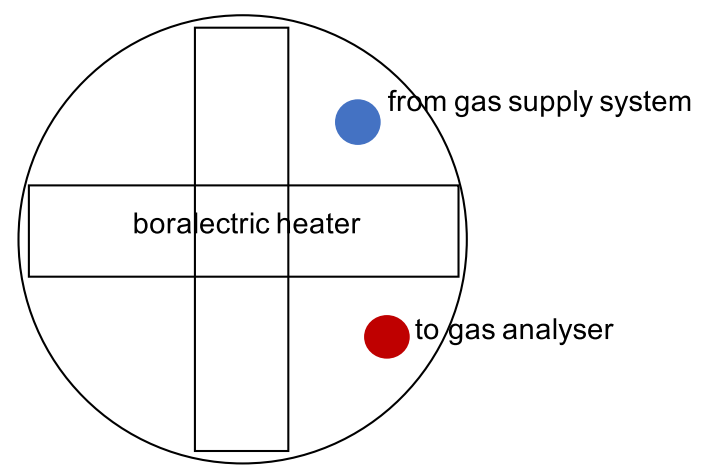

FIG. 1. (a) Cross section of the Leiden probe microscopy mini flow reactor. A transparent window closes the reactor from the top. The sample is placed on top of the boralectric heater that can heat the sample up to $800{ }^{\circ} \mathrm{C}$. With a thermocouple attached to the sample, we measure the temperature. The feedthroughs for the gases, heater, and the thermocouple are below the sample holder in the bottom plate. Gas flows in from the gas supply system (blue arrow) and out towards the gas analyzer (red arrow). The sample holder and reactor enclosure are similar to those of the ReactorSXRD chamber ${ }^{19}$ and can be exchanged. (b) Schematic top view of the reactor showing the boralectric heater, the position of the gas inlet, and the position of the gas outlet. 


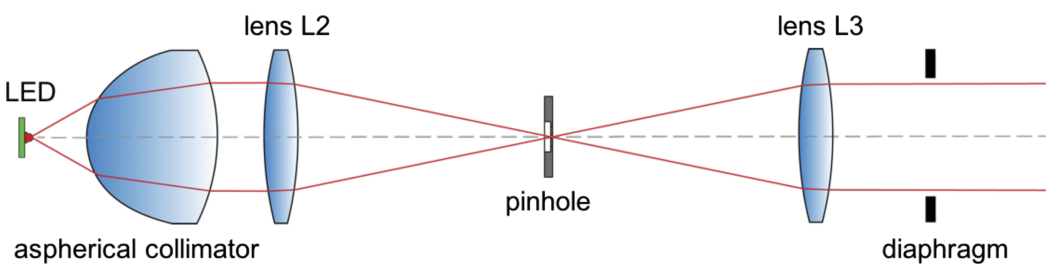

FIG. 2. Schematic design of the light source and the spatial filter. Light from a high-intensity LED is collected using an aspherical collimator. A second lens focuses the beam onto the pinhole P. A third lens (L3) with the focal point located at the position of the pinhole $\mathrm{P}$ creates a parallel beam of light. With a diaphragm, we match the size of the beam to the sample size.
As a light source, we use a Thorlabs M625L3 LED with a $625 \mathrm{~nm}$ central wavelength. The light is first collimated after the source with an aspherical lens with a $40 \mathrm{~mm}$ focal length and a numerical aperture (NA) of 0.554 to capture a large fraction of the emitted light. The light passes a diffusor to eliminate any spatial pattern arising from the light source, then the light is spatially filtered with a lens with $10 \mathrm{~cm}$ focal length that focuses the light on a $150 \mu \mathrm{m}$ pinhole. After the pinhole, a lens with $6 \mathrm{~cm}$ focal length collimates the beam, recreating a parallel beam. A diaphragm after the spatial filter allows us to set the beam diameter. We do not measure the LED intensity and therefore cannot correct the measured data for fluctuations in the light. A schematic design of the light source and spatial filter is shown in Figure 2.

A pellicle beam splitter reflects $50 \%$ of the light towards the sample. $50 \%$ of the light that is reflected by the sample passes through the beam splitter towards the camera. Behind the beam splitter, the post-sample stage starts. Figure 3 shows four different lens configurations that can be applied to disentangle the different reflected components. In Figure 3(a), all reflected light is used to create an image of the sample. Figure 3 (b) corresponds to a bright-field configuration where we use a pinhole in the focal plane of lens L4, in order to select only the specularly reflected components. In the dark-field configuration (Figure 3(c)), a beam stop is placed in the focal plane of lens L4. In this way, the specularly reflected beam is blocked and only the light that is diffusely scattered from the sample is allowed to pass. L5 behind the beam stop focuses the remaining light on the camera. In Figure 3(d), the camera is placed in the focal plane of L4. This creates a far-field image that registers the angles at which the light is reflected at the surface. In principle, the optical system could have been designed such, that it integrated various imaging modes in the same setup. For simplicity, we chose to simply reconfigure the optical setup

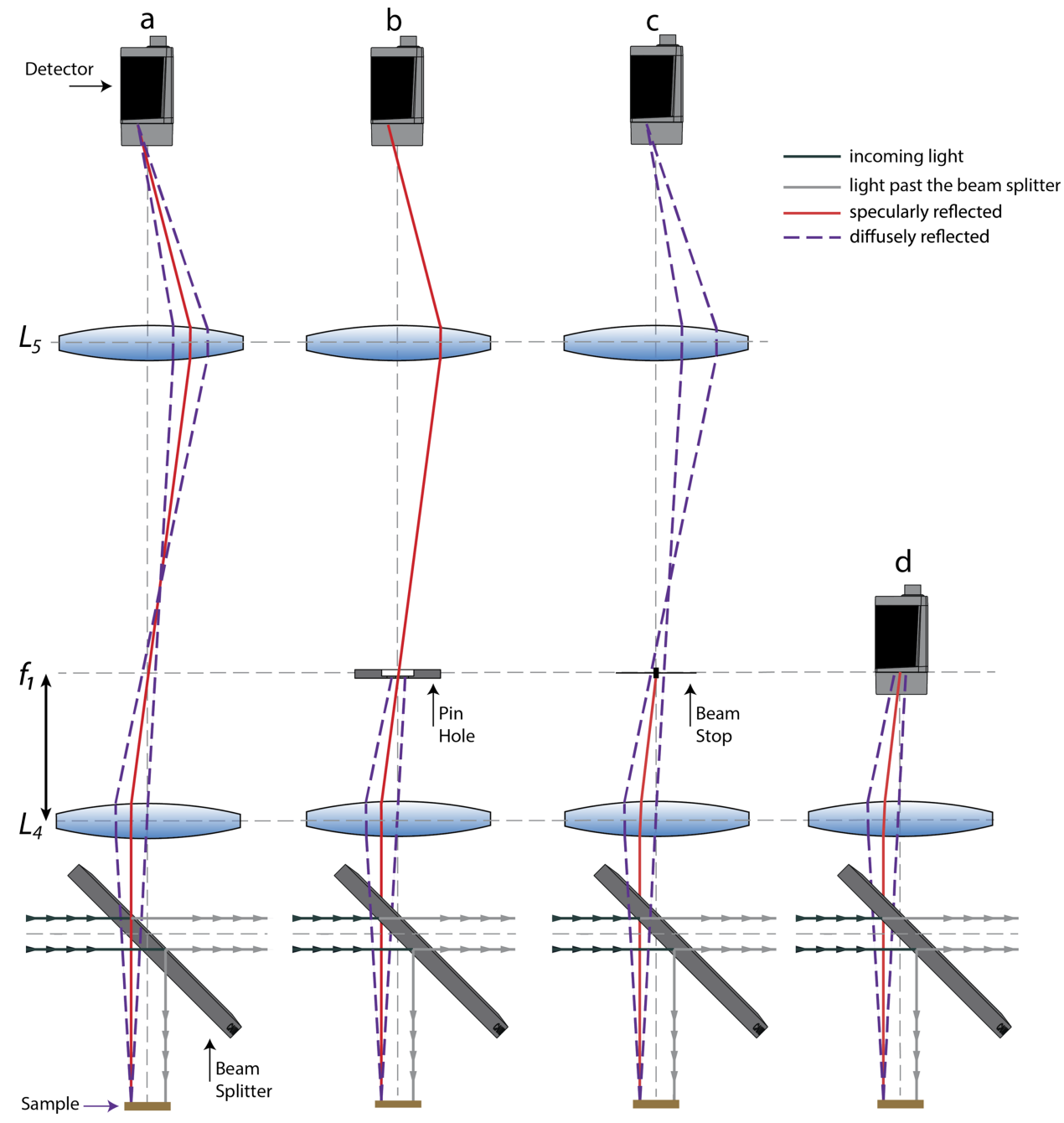

FIG. 3. Schematics of several possible configurations of the optical system. A parallel light beam arrives from the left where it hits the beam splitter. Half of the intensity is reflected downwards and half is transmitted. After reflection on the sample surface (gold), the light is transmitted through the beam splitter. Four analysis methods are indicated. (a) Imaging of the surface. (b) In a bright-field configuration, diffusely reflected light (purple) is blocked by the pinhole. (c) In a dark-field configuration, specularly reflected light (red) is blocked by the beam stop. (d) In an angular configuration, the distance to the center of the camera is determined by the angle of scattering. 
between different types of experiments. Since the illumination stage is identical, data collected in different configurations can be directly compared. In this paper, we will only show results of the bright-field reflectivity mode.

To record the images, we use a Basler acA2500-14gm CCD camera. This camera is a monochrome camera with 12 bits per pixel and has a resolution of $2592 \times 1944$ pixels. The camera is fully computer controllable, and parameters such as pixel gain, pixel binning, integration time, and time between frames can be adjusted. The maximum frame rate of the camera is 14 frames/s.

The sample in the reactor is directly mounted on a boralectric heater, a graphite heating element embedded in boron nitride. This heater is connected to a Delta ES 030-5 power supply and can be heated to $800^{\circ} \mathrm{C}$, the window of the optics part being the limiting factor. The sample holder carrying the boralectric heater is mounted on two tantalum rods that are connected to a ceramic unit. Thermocouple wires connected to pins in the sample holder are (laser)-spot-welded on the sample to measure the temperature. The pins underneath the sample holder connect to sockets in the reactor base. The sockets are connected to feedthroughs connecting the signal to the outside of the reactor. The power supply as well as the thermocouple is computer readable and controlled. Feedback is applied between temperature readout and heating power, in order to keep the temperature of the sample constant.

\section{PERFORMANCE, FIRST RESULTS, AND DISCUSSION}

In this section, we demonstrate the sensitivity of the optical reflection technique by applying it to the (100) surface of palladium, subjected to $\mathrm{CO}$ and $\mathrm{O}_{2}$ gas at high temperature and near-atmospheric pressure. We will see that, even though the surface changes appear modest, a clear change can be observed in the optical reflection signal.

Figure 4 shows the first results obtained with our newly developed optical setup. We monitor the relative reflectance difference $\Delta \mathrm{R}$ of the $\mathrm{Pd}(100)$ sample during $\mathrm{CO}$ oxidation. Here we define $\Delta R=\left(I-I_{0}\right) / I_{0}$ with $I_{0}$ the reflected intensity

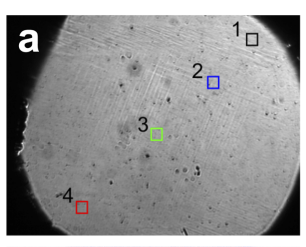

b
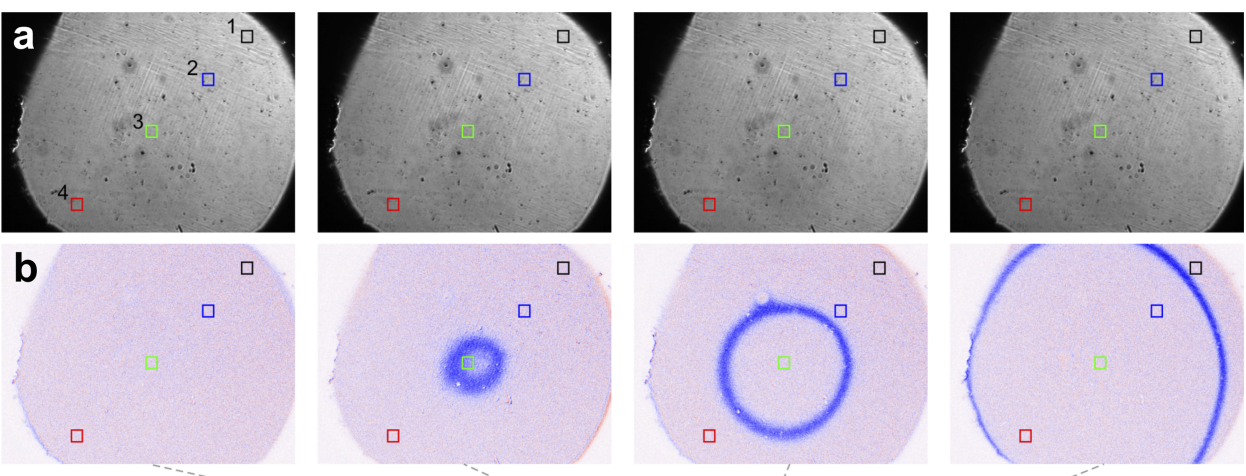

C

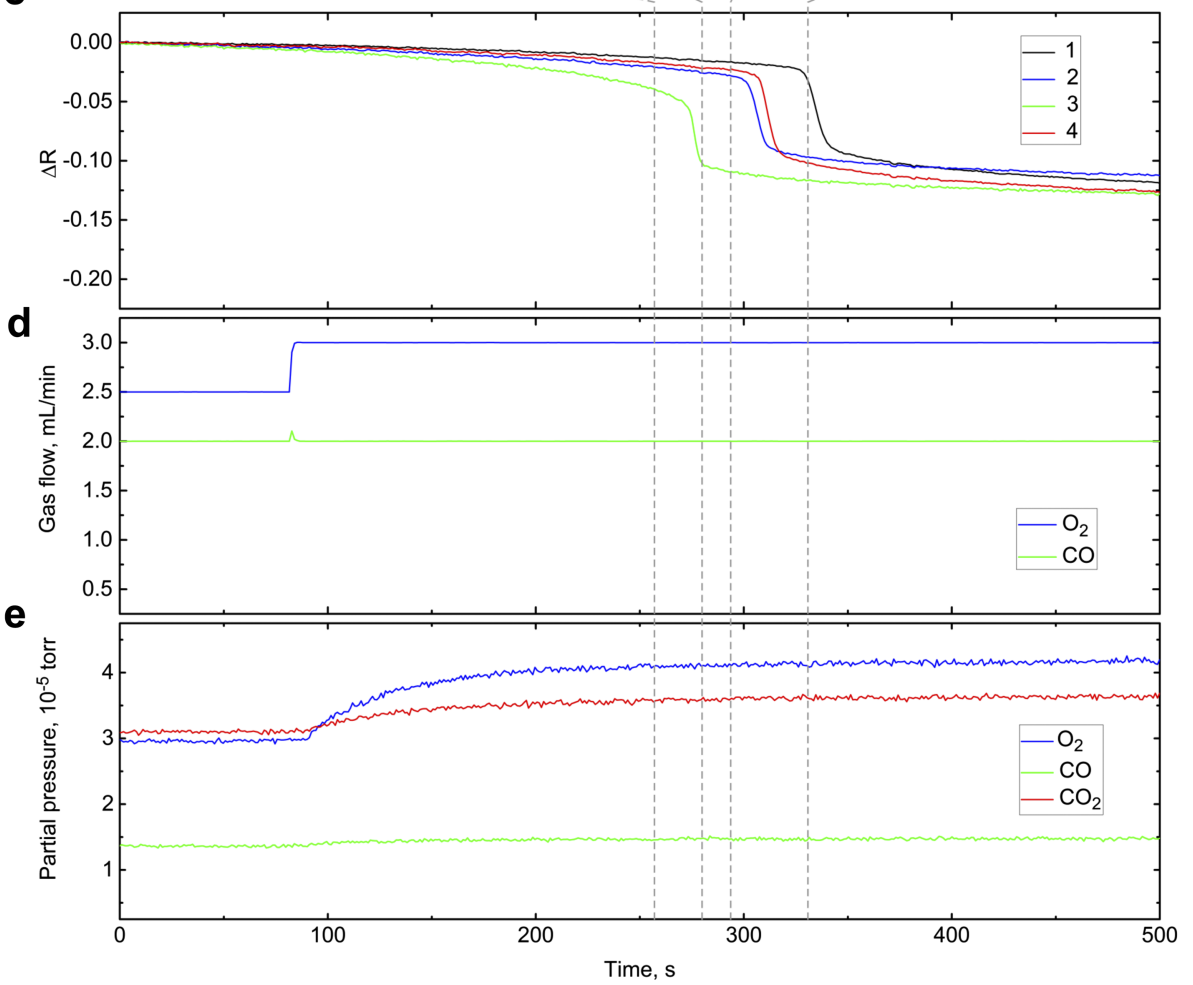

FIG. 4. (a) Direct output of the CCD camera at four moments in time, as indicated by the vertical dashed grey lines. (b) Output of the CCD camera differentiated over time, i.e., the slope of light intensity was calculated by linear regression from 5 adjacent frames, with the current frame in the middle, and colorenhanced: red is an increase in reflectivity and blue is a decrease in reflectivity. (c) Variation of reflectivity $\Delta \mathrm{R}$ with time. The data for the reflectivity curves are taken from the areas indicated by squares in Figs. 4(a) and 4(b). (d) Flow rates at which $\mathrm{CO}$ and $\mathrm{O}_{2}$ have been introduced into the reactor. (e) Composition of the gas leaving the reactor as measured by the residual gas analyzer. 
at the start of the experiment and $I$ the measured intensity at later stages of the experiment. Prior to the experiment, the sample was reduced in a flow of pure $\mathrm{CO}$ at $320^{\circ} \mathrm{C}$ to ensure that the surface was in the metallic state, resulting in a highly reflective surface (not shown). Following this reduction procedure, the $\mathrm{Pd}(100)$ surface was exposed to a $\mathrm{CO} / \mathrm{O}_{2}$ gas mixture at $300 \mathrm{mbar}$ and $320^{\circ} \mathrm{C}$. The $\mathrm{CO}$ flow was kept constant at $2 \mathrm{ml}_{n} / \mathrm{min}$, while the $\mathrm{O}_{2}$ flow was increased from 2.5 to $3 \mathrm{ml}_{n} / \mathrm{min}$ (see panel (d) of Figure 4).

Figure 4(a) shows the direct CCD camera output of the sample using the bright-field mode (see lens configuration (b) in Figure 3). The four images are taken from the full captured movie at times corresponding to the vertical dashed grey lines (i.e., $t=257,280,294$, and $331 \mathrm{~s}$ ). Figure 4(b) shows the same images but differentiated in time, i.e., the slope of light intensity was calculated by linear regression from 5 adjacent frames, with the current frame in the middle. For clarity, a $\mathrm{red} /$ white/blue color scale is used, where red corresponds to a reflectivity increase and blue corresponds to a reflectivity decrease. From the full sequence of images, we extract the $\Delta \mathrm{R}$ curves with the behavior of the sample over time. We do this by integrating a small area of the sample for each of the images (see the squares in Figs. 4(a) and 4(b)), which results in $I(t)$. From this, we calculate $\Delta \mathrm{R}$ (Figure 4(c)). In Figures 4(d) and 4(e), we show the output of the mass flow controllers and of the high pressure inlet residual gas analyzer, respectively.

When exposing the $\mathrm{Pd}(100)$ surface to a mixture of $\mathrm{CO}$ and $\mathrm{O}_{2}$ (2:2.5 ratio), we observe that the reflectivity of the sample slowly decreases (see $t=0 \mathrm{~s}$ to $\mathrm{t}=81 \mathrm{~s}$ in panel (c) of Figure 4). When increasing the amount of oxygen present in the gas mixture from $2.5 \mathrm{ml}_{n} / \mathrm{min}$ to $3 \mathrm{ml}_{n} / \mathrm{min}$ (at $\mathrm{t}=81 \mathrm{~s}$ ), the reflectivity further decreases, albeit at a faster pace. The first two images of panels (a) and (b) show the direct output of the CCD camera and the differentiated image at $\mathrm{t}=257$ and $280 \mathrm{~s}$. From SXRD experiments and STM observations, we know that the $\operatorname{Pd}(100)$ surface forms a thin surface oxide under oxidizing conditions. ${ }^{4,6}$ Therefore, we attribute the initial moderate decrease in reflectivity to the formation of a thin surface oxide. From analysis of all images, it can be concluded that the decrease of $\Delta \mathrm{R}$ due to the presence of an oxidizing atmosphere in the reactor is first observed closest to the gas inlet (see Fig. 1(b) for the exact location of the gas inlet). The same is observed for reduction of the surface when introducing $\mathrm{CO}$ in the reactor (not shown). When we increase the partial pressure of $\mathrm{O}_{2}$ in the gas mixture, the $\mathrm{CO}_{2}$ production slowly increases (see Figure 4(e)). The small increase in CO partial pressure is due to the presence of $\mathrm{CO}$ and $\mathrm{N}_{2}$ in $\mathrm{O}_{2}$ as contaminants (with the residual gas analyzer, we cannot distinguish between $\mathrm{CO}$ and $\mathrm{N}_{2}$ ). During exposure to the more oxygen-rich gas mixture, at $\mathrm{t}=280 \mathrm{~s}$, we observe a darkening of the sample (Fig. 4(a)) and a corresponding steep drop in the reflectivity (Fig. 4(c)). In the differentiated image in panel (b), this is visible as the formation of a blue ring at the surface. We attribute this decrease in reflectivity to the formation of a thicker surface oxide, similar to what we observed before in SXRD and STM. ${ }^{4,6}$ The darkening of the sample is slightly delayed ( $\sim 10 \mathrm{~s})$ compared to the moment that we increase the partial pressure of $\mathrm{O}_{2}$ in the gas mixture. This is due to the time it takes for the gases to reach the gas analyzer including the diffusion through the reactor $(0.6 \mathrm{ml}$ gas lines with a flow rate of $16.7 \mathrm{ml} / \mathrm{min}$ correspond to $\sim 2.2 \mathrm{~s}$, additional time results from the flow through the reactor). As can be seen from the images in panels (a) and (b) of Figure 4, the formation of the thicker surface oxide starts from the middle of the Pd(100) surface and spreads in concentric circles over the entire sample. The last images show a surface that is almost completely covered by the thicker surface oxide. We attribute this formation of oxide from the middle of the surface to the fact that the center of the sample has the highest temperature, since the heating by the boralectric heater is mostly concentrated there. When increasing the oxygen flow, we observe a decrease in heating power needed to keep the sample at a constant temperature of $320^{\circ} \mathrm{C}$. This decrease in heating power is due to the increased production of $\mathrm{CO}_{2}$ via $\mathrm{CO}$ oxidation, which is an exothermic reaction. The observed fluctuations in the heating power follow the observed behavior in $\mathrm{CO}_{2}$ production. The formation of the surface oxides is reversible: When we remove the oxygen from the gas mixture, the sample becomes shiny again and the initial reflectivity value is restored, indicating that the surface has become smooth, i.e., metallic, again. This reduction of the surface is fast and causes a sharp jump (increase) in the observed reflectivity. We will discuss the distinction between surface roughness and oxide formation as the possible origins for the observed changes in the reflectivity in a forthcoming paper.

\section{CONCLUSIONS}

In this paper, we present a new method for performing in situ investigations of model catalysts under reaction conditions of atmospheric pressures and elevated temperatures. We are able to follow the optical response of a catalyst surface to a range of gas conditions. The technique is sensitive to small changes in reflectivity of the catalyst surface. We use a setup that is far less complex, yet complementary to the SXRD ${ }^{19}$ and STM $^{21}$ instruments previously used, making it possible to link the macroscopic scale behavior of the active catalytic surface to the microscopic observations. Therefore, a large range of catalytic environments can be easily monitored, and this technique is a valuable addition to the existing methods available for investigating high-pressure catalysis in situ. The roughening of the $\operatorname{Pd}(100)$ surface due to formation of a surface oxide during CO oxidation, as previously observed with STM and SXRD, ${ }^{4,6}$ was corroborated using this novel optical method.

\section{ACKNOWLEDGMENTS}

This work was financially supported through Grant No. 12825 of Stichting Technologische Wetenschappen (STW) of the Netherlands Organization for Scientific Research (NWO). I.M.N.G. acknowledges support through a Veni grant of STW. The authors thank Bart Nelissen (Albemarle) and Joachim Loos (DSM Resolve) for fruitful discussions.

\footnotetext{
${ }^{1}$ E. H. Conrad and T. Engel, Surf. Sci. 299-300, 391 (1994).

${ }^{2}$ M. S. Hoogeman, M. A. J. Klik, D. C. Schlößer, L. Kuipers, and J. W. M. Frenken, Phys. Rev. Lett. 82, 1728 (1999).

${ }^{3}$ Operando Research in Heterogeneous Catalysis, edited by I. M. N. Groot and J. W. M. Frenken (Springer Verlag, 2017).
} 
${ }^{4}$ B. L. M. Hendriksen, S. C. Bobaru, and J. W. M. Frenken, Surf. Sci. 552, 229 (2004).

${ }^{5}$ R. van Rijn, O. Balmes, A. Resta, D. Wermeille, R. Westerstrom, J. Gustafson, R. Felici, E. Lundgren, and J. W. M. Frenken, Phys. Chem. Chem. Phys. 13, 13167 (2011).

${ }^{6}$ E. Lundgren, J. Gustafson, A. Mikkelsen, J. N. Andersen, A. Stierle, H. Dosch, M. Todorova, J. Rogal, K. Reuter, and M. Scheffler, Phys. Rev. Lett. 92, 046101 (2004).

${ }^{7}$ B. L. M. Hendriksen, M. D. Ackermann, R. van Rijn, D. Stoltz, I. Popa, O. Balmes, A. Resta, D. Wermeille, R. Felici, S. Ferrer, and J. W. M. Frenken, Nat. Chem. 2, 730 (2010).

${ }^{8}$ J. Gustafson, M. Shipilin, C. Zhang, A. Stierle, U. Hejral, U. Ruett, O. Gutowski, P. A. Carlsson, M. Skoglundh, and E. Lundgren, Science 343, 758 (2014).

${ }^{9}$ B. L. M. Hendriksen, S. C. Bobaru, and J. W. M. Frenken, Top. Catal. 36, 43 (2005).

${ }^{10}$ B. L. M. Hendriksen, S. C. Bobaru, and J. W. M. Frenken, Catal. Today 105, 234 (2005).
${ }^{11}$ P. Mars and D. W. van Krevelen, Chem. Eng. Sci. 3, 41 (1954).

${ }^{12}$ S. Matera and K. Reuter, J. Catal. 295, 261 (2012).

${ }^{13} \mathrm{~S}$. Nettesheim, A. von Oertzen, H. H. Rotermund, and G. Ertl, J. Chem. Phys. 98, 9977 (1993).

${ }^{14}$ D. E. Aspnes, Surf. Sci. 307-309, 1017 (1994).

${ }^{15}$ Y. Borensztein, Phys. A 207, 293 (1994).

${ }^{16}$ H. H. Rotermund, Surf. Sci. Rep. 29, 265 (1997)

${ }^{17}$ H. H. Rotermund, Surf. Sci. 603, 1662 (2009).

${ }^{18}$ J. Dicke, P. Erichsen, J. Wolff, and H. H. Rotermund, Surf. Sci. 462, 90 (2000).

${ }^{19}$ R. van Rijn, M. D. Ackermann, O. Balmes, T. Dufrane, A. Geluk, H. Gonzalez, H. Isern, E. de Kuyper, L. Petit, V. A. Sole, D. Wermeille, R. Felici, and J. W. M. Frenken, Rev. Sci. Instrum. 81, 014101 (2010).

${ }^{20}$ See http://www.leidenprobemicroscopy.com/ for Leiden Probe Microscopy B.V.

${ }^{21}$ P. B. Rasmussen, B. L. M. Hendriksen, H. Zeijlemaker, H. G. Ficke, and J. W. M. Frenken, Rev. Sci. Instrum. 69, 3879 (1998). 